\title{
Disruption Management Recovery Model of Distribution Delay with Service Priority
}

\author{
Li Jiang ${ }^{1}$, Hongyan Wang ${ }^{1} \&$ Bin Ding $^{2}$ \\ ${ }^{1}$ School of Management, Hefei University of Technology, Hefei, China \\ ${ }^{2}$ School of Management, University of Science \& Technology of China, Hefei, China \\ Correspondence: Li Jiang, School of Management, Hefei University of Technology, Hefei, Anhui, 230009, \\ China. Tel: 86-551-6291-9151. E-mail: jianglix@mail.ustc.edu.cn
}

Received: November 22, 2012 Accepted: December 21, 2012 Online Published: January 28, 2013

doi:10.5539/ass.v9n2p170

URL: http://dx.doi.org/10.5539/ass.v9n2p170

\begin{abstract}
During the process logistics distribution, distribution delay would decrease customer service level, some customers especially key customers will take some new suppliers with higher service level to replace the existing suppliers, and this change will brings huge risk even great loss to the origin suppliers. To help suppliers success to keep old key customers and reduce their risk of key customer loss, this study brings disruption management idea into vehicle routing problem (VRP), presents a delivery service priority method which considers customers' service priority and provide prior delivery service to key customers in their time window. A single-vehicle delivery delay disruption management recovery model with service priority and a multi-vehicle delivery delay disruption management recovery model with service priority are constructed to ensure all the key customers' service level when distribution delay occurred. Then, an improved Genetic algorithm is designed to solve them. Finally, a simulation example model is presented to demonstrate the feasibility and validity of the proposed models and algorithm. This study provides two referenced models and solution algorithm for disruption management of logistics distribution network composed by one supplier (or a third-party logistics company) and multiple customers.
\end{abstract}

Keyword: disruption management, distribution delay, vehicle routing problems (VRP), service priority, genetic algorithm

\section{Introduction}

In recent years, with the developing of supply chain management, distribution model of multi-varieties, small batch and just in time is commonly applied to adapt the "inventory less management" in each node of supply chain. However, traffic congestion, vehicle failure, road maintenance and other disruption factors are prone to lead to delivery delay (Zeng, 2012). These disruption incidents will weaken logistics management efficiency of multi-varieties, small batch and just in time, even will increase the operation cost of supply chain. To solve distribution delay problem, scholars at home and abroad have done a lot of researches. Hiusmanet et al. (2004) study the multi-depots vehicle scheduling problem with travel time delay. In order to minimize vehicle number, percentage of customer service delay, delay cost, they convert multi-depots scheduling problem into single-depot scheduling problem with the clustering-rearranging algorithm. Woensel (2008) uses a method based on the queue theory to solve the emergency scheduling problem of the vehicle traffic jams. The experiment result illustrates that this method could save transportation time. Zeimpekis et al. (2005) study disruption problem on distribution delay and vehicle failure, take customers' requirements of the time window restriction into consideration, design a management system to solve these problems with the objective of minimizing the distribution delay cost and maximizing the number of serviced customers. Zhang Y H (2007) sets traffic jam points as virtual customers, a mathematic model is constructed with the objective of minimizing vehicle numbers and distribution time to solve emergency vehicles scheduling problem on traffic jam. Zhang Y. (2007) constructs a mathematic model with the objective of minimizing the distribution cost and system disruption to solve distribution delay problem with hard time window. As for the problem of decreasing service level caused by distribution delay, Zhang Q. (2008) takes precondition that the total distribution delay time must meet preset value into consideration, constructs a single-vehicle disruption management model on distribution delay and uses genetic algorithm to solve the model.

All the above literatures have the same basic assumption. Suppliers (or logistics providers) treat all the customers 
without discrimination. It means that all the customers are serviced on an equal footing in any situation. However, according to the theorem of $20 / 80,80 \%$ profit of the enterprise comes from $20 \%$ of the key customers, thus, it's very important for enterprises to improve service level for key customers and maintain good relations with them at any time. On the basis of the above 20/80 theorem and practical experience, we found that keep key customer is the key to success for suppliers, especially in some emergency situation. This study assumes that different customer has different service priority level. It means that key customers with higher priority will be serviced prior to others in their time windows when distribution delay occurred. This study uses disruption management idea to construct two distribution delay recovery models with service priority. The objectives are also to minimize the distribution cost, the routs change and the total distribution delay with service priority, and they will be called under different situation. The principle of calling models is to ensure the service level of the $20 \%$ key customers when distribution delay occurred, at the same time, reduce total distribution cost to a lowest level.

\section{Vehicle Routing Problems (VRP) and Disruption Management (DM)}

Dantzig and Ramser (1959) first puts forward the vehicle routing problem (vehicle routing problems, VRP), which can be described as follows. Distribution center deliver goods to a certain amount customers with different demands. On the premise of satisfying the needs of customers, arrange optimal vehicle routes to ensure the shortest distribution distance, lowest cost and the least time consuming, etc. On the research basis of VRP, there are so many stretches on VRP, such as vehicle routing problems with time windows constraint, VRPTW (1996), fleet size and mix vehicle routing problems, FSVRP (1984), vehicle routing problems with multiple use of vehicle, VRPM (1996), vehicle routing problems with backhauls, VRPB (1989), vehicle routing problems with stochastic demand, VRPSD (1989), and so on. In a word, there are two kinds of vehicle routing problems with time windows which are vehicle routing problems with hard time windows constraint and vehicle routing problems with soft time windows constraint. VRP with hard time window constraint is means that customers' time window requirement cannot be violated. If the distribution vehicle arrives at the customer point before the earliest permission time to service, thus, need to wait until the customers' time window. If the distribution vehicle arrives the customer point after the last permission time to service, it will be set for an infinite punishment coefficient. In addition, regards to VRP of soft time window constraint, customers' time window requirement is allow to be violated in a certain range. If the distribution vehicle arrives at the customer point before the earliest permission time to service, thus, also need to wait until the customers' time window. If the distribution vehicle arrives the customer point after the last permission time to service, it will be set for a punishment coefficient which is proportional to distribution delay time.

Vehicle routing problem (VRP) could ensure the shortest distribution distance, minimum distribution cost through some optimization goal set. However, it also has some deficiencies.

1) Vehicle routing problem (VRP) is statics rout reschedule.

2) The emergency response plan is not flexible enough to deal with the changeful actual situation about distribution.

3) Reschedule all the routes, then, the extensive adjustment of the original route will make its optimal solutions infeasible.

Disruption Management is to deal with some usually disruption accidents, such as production equipment failure, distribution vehicle failure, traffic jams, demand change of supply chain, and so on. At present, there is no unified definition about disruption management in academics. However, all the scholars both at home and abroad have been achieved a consensus effectively. Yu Gang (2004), American scholar, defines disruption management as follows. In early stage, a good operational plan can be got through some optimization model and algorithm. However, in the stage of implementing the plan, due to internal and external uncertain elements, the original plan may become infeasible and a new real-time plan needs to be generated. The new real-time plan must take the original optimization objective into account, and to minimize the negative effects at the same time. Clausen (2001) also applies disruption management idea to make variation return to original plan, so as to minimize the system disruption. Chinese scholars, An Chen (2006), holds that disruption management is a management approach that can make the interfere incident back to its original state. It mainly focuses on incidents that slightly deviate from the original plan. While deviation is slight and will not cause severe negative impact, some active management method can be used to correct it. So, to summarize, we think that disruption management should include several basic elements.

1) Original Plan. Plan that system under normal operation or the best plan that considers various factors in normal state. 
2) Disruption incident. Incident that affects system operation, for example, vehicle's breakdown, customers demand changes, and so on. Incident in different areas may be different, but all the incidents must be occurred frequently and randomly.

3) Recovery model. When disruption incident occurred, adjust the original distribution rules and construct recovery model. Different disruption incident usually has different optimization model. In the same way, the model can be constructed with multiple optimization objectives. However, it must contain the goal of minimizing the impact degree of the original plan. While specific to distribution area, the goal may be the minimum impact of suppliers or customers;

4) Algorithm to solve the recovery model. Algorithm can use exact solution or approximate solution according to specific problems, but the algorithm has a basic requirement that is quick to solve the model, so as to respond quickly when disruption incident occurred.

Furthermore, we find it is good to improve the above deficiencies about VRP, if the thought of disruption management were applied to the vehicle routing problem (Wang X P, 2008; G. M. Giaglis, 2004; Yang W Z, 2010).

1) Disruption management is to deal with usually disruption accidents, which could adjust route in a dynamic way.

2) Adjust the route in a dynamic and real-time way, it has a strong point.

3) Optimal solution is feasible, because one of the optimization objectives is to minimize route adjustment of the original scheme.

\section{Distribution Delay Recovery Model Based on Disruption Management}

This study, the idea of disruption management is applied to solve the vehicle routing problem, construct a single-vehicle delivery delay disruption management recovery model with service priority and a multi-vehicle delivery delay disruption management recovery model with service priority. And the objectives are both to minimize distribution delay time, distribution cost and route change. In order to reduce disruption degree for the original plan, the detailed model calling rule can be described as follows. When disruption incidents happened, give priority to call single-vehicle disruption management model, only to adjust customer service points which belong to the vehicle route that is delay. But if the single-vehicle disruption management model couldn't guarantee all the key customers' logistics service level, call the multi-vehicle disruption management to adjust more distribution vehicle routes.

\subsection{Problem Description}

In a distribution network composed of one supplier (or the third party logistics enterprise) and many distribution customers, all the customers' service priorities are distinguished according to their property and importance. Suppliers (or the third party logistics enterprises) could get their distribution routes with the objective of minimizing distribution distance and meeting customers' time window requirement. A normal distribution, suppliers arrange distribution routes according to the original scheme, and all the customers have the same priorities. But if the distribution delay happened because of vehicle traffic congestion or vehicle broken down (repair time is short), customers' distribution service priorities will begin to be distinguished in the follow distribution. That is to say, the customer with higher distribution service priority is prior to be served in its time window. This study we use soft time windows restrictions. Customer will choose not to cancel the distribution service but to take measures to punish the suppliers, on condition that vehicles arrive at customer point beyond their time windows.

\subsection{Disruption Management Recovery Model of Distribution Delay with Service Priority}

This section we provide mathematical model of the distribution delay with service.

We make some assumptions as follows.

1) When vehicle failure happened, it is easy to get the follow information in real time, such as vehicle's coordinate, vehicle's loading capacity, customers that have been served and not to be served, and so on.

2) Traffic congestion and vehicle failure will lead to distribution delay, and the distribution delay time can be estimated accurately.

3) Ignore the different of route direction, that is to say, the route A to B in the original scheme is equivalent to the route of $B$ to $A$ in the new scheme. This is regarded as no change in the new scheme.

We denote the parameters by: 
$X$ : initial optimal route set;

$X^{*}$ : optimal route set after disruption recovery;

$c_{i j}$ : distribution cost of the vehicle form customer i to customer $\mathrm{j}$;

$d_{i}$ : demand of customer i;

$v$ : total number of the distribution vehicles;

$q_{k}$ : loading capacity of the vehicle $\mathrm{k}$;

$t_{i j}$ : vehicle travel time from customer i to customer $\mathrm{j}$;

$t_{i}$ : vehicle service time in customer i;

$s t_{i}$ : start time when the vehicle arrive at customer i and begin to serve for it;

$\left[l t_{i}, h t_{i}\right]$ : distribution service time window of customer i;

$a^{+}$: side set which belong to the original route set of $X$, but don't belong to the adjusted route set of $X^{*}$;

$a^{-}$: side set which belong to the adjusted route set of $X^{*}$, but don't belong to the original route set of $X$;

$\lambda$ : deviation cost that delete one side in the original route set;

$\lambda^{\prime}$ : deviation cost that add a new side to the new route set;

$w_{i}$ : distribution delay priority of customer $\mathrm{i}$.

We described the follow two Variables:

$$
x_{i j}=\left\{\begin{array}{ll}
1 & i \rightarrow \mathrm{j} \\
0 \mathrm{i} \rightarrow \mathrm{j}
\end{array} \quad x_{i j k}= \begin{cases}1 & i, k \rightarrow \mathrm{j}, \mathrm{k} \\
0 & \mathrm{i}, \mathrm{k} \rightarrow \mathrm{j}, \mathrm{k}\end{cases}\right.
$$

The overall mathematical model is as follows:

(1) Single-vehicle disruption recovery model of distribution delay with service priority

$$
\begin{aligned}
& o b j=\min \left[\sum_{\forall i, j} c_{i j} x_{i j}+g\left(a^{+}, a^{-}\right)+\sum_{\forall i} w_{i} \text { ltime }_{i}\right] \\
& g\left(a^{+}, a^{-}\right)=\lambda\left|a^{+}\right|+\lambda^{\prime}\left|a^{-}\right| \\
& \text {s.t. }\left\{\begin{array}{l}
\sum_{j} x_{i j}=1 \forall i \\
\sum_{i} x_{i h}=\sum_{j} x_{h j} \forall h \\
s t_{i}+t_{i}+t_{i j}-M\left(1-x_{i j}\right) \leq s t_{j} \forall i, j \\
l t i m e_{i}=\max \left(s t_{i}-h t_{i}, 0\right) \forall i \\
X+a^{+}-a^{-}=X^{*} \\
x_{i j}=\{1,0\}
\end{array}\right.
\end{aligned}
$$

The objective of this model is to minimize distribution delay time with service priority, distribution cost and route change. Formulation (1) indicates the adjusted cost of the new scheme relative to the original scheme. Constraint (2) and Constraint (3) guarantees that one customer is serviced by only one vehicle. Constraint (4) express that arrival time of the vehicle to customer $\mathrm{j}$ composed of the arrival time to customer $\mathrm{i}$, service time for customer $\mathrm{i}$ and travel time from customer $\mathrm{i}$ to customer $\mathrm{j}$. Constraint (5) is the distribution delay time when vehicle arrive at customer i. If vehicle arrive at customer i before $l t_{i}$, it must wait until $l t_{i}$ to begin to serve, and then, ltime $_{i}=0$, If vehicle arrive at customer $\mathrm{i}$ after $l t_{i}$, then, ltime $e_{i}>0$. Constraint (6) shows that the total routes of the original scheme are same as the new scheme.

(2) Multi-vehicle disruption recovery model of distribution delay with service priority 


$$
\begin{aligned}
& o b j=\min \left[\sum_{\forall i, j, k} c_{i j} x_{i j k}+g\left(a^{+}, a^{-}\right)+\sum_{\forall i} w_{i} \text { ltime }_{i}\right] \\
& g\left(a^{+}, a^{-}\right)=\lambda\left|a^{+}\right|+\lambda^{\prime}\left|a^{-}\right| \\
& \text {s.t. }\left\{\begin{array}{l}
\sum_{j} \sum_{k} x_{i j k}=1 \forall i \\
\sum_{i} x_{i h k}-\sum_{j} x_{h j k}=0 \forall h, k \\
\sum_{i, k} x_{0 i k}=v \\
\sum_{i}\left(d_{i} \sum_{j} x_{i j k}\right) \leq q_{k} \forall k \\
s t_{i}+t_{i}+t_{i j}-M\left(1-\sum_{k} x_{i j k}\right) \leq s t_{j} \forall i, j \\
l t i m e_{i}=\max \left(s t_{i}-h t_{i}, 0\right) \forall i \\
X+a^{+}-a^{-}=X \\
x_{i j k}=\{0,1\}
\end{array}\right.
\end{aligned}
$$

This model also has the objective of minimizing distribution delay time with service priority, distribution cost and route change Constraint (7), Constraint (8) and Constraint (9) show that each of the customers should be served by the only one vehicle. Constraint (10) indicates that customers' demand that each vehicle served for must be less than its loading capacity. Constraint (11) express that arrival time of vehicle $\mathrm{k}$ to customer $\mathrm{j}$ composed of the arrival time to customer $\mathrm{i}$, service time for customer $\mathrm{i}$ and travel time from customer $\mathrm{i}$ to customer j. Constraint (12) is the distribution delay time when vehicle arrive at customer i. If vehicle arrive at customer $\mathrm{i}$ before $l t_{i}$, it must wait until $l t_{i}$ to begin to serve, and then, ltime $e_{i}=0$, If vehicle arrive at customer $\mathrm{i}$ after $l t_{i}$, then, ltime $_{i}>0$.Constraint (13) shows that the total routes of the original scheme are same as the new scheme.

\section{The Solution for the Distribution Management Recovery Model of Distribution Delay}

This study, we design an improved Genetic Algorithm to solve above models. As a Heuristic Algorithm, it could get optimal scheme quickly. The progress of the algorithm can is described as follows.

\subsection{Encoding}

To reduce program complexity, we take one dimensional coding which can be described as follows. If a chromosome path is 4-3-2-1-5-6-7-8-9, it will be cut into several sections according to each vehicle's loading capacity, and vehicles will be arranged to serve for customers by these chromosome sections. It means to accumulate customers' demand from starting point according to chromosome order until the total demands reaching vehicle loading capacity, and then arrange the next vehicle from the end of the first chromosome section. For Example, if

$$
\sum_{i=1}^{4} d_{i} \leq q_{1} \text { and } \sum_{i=1}^{5} d_{i}>q_{1},
$$

then, the route of the first vehicle is 4-3-2-1. As this way, other vehicles' routes can be easy to get. At last, calculate the vehicle numbers that the individual chromosome needs. If the vehicle number is $\mathrm{K}$, and if $K>v$, then, this chromosome is unfeasible solution. However, if $K \leq v$, this chromosome is feasible solution.

\subsection{Fitness Function}

Fitness function is the evaluating basis of individual quality of genetic algorithm, the individual with higher fitness would be trained prior. As for this problem, assume that the population number of chromosome is N. the smaller Chromosome fitness value, the higher individual fitness value. We set the fitness function as the countdown of the objective function values which can be described as $f=1 /$ obj $_{i}$.

\subsection{Select}

Population group is selected and copied by the selection operator which is direct to individual fitness value. The probability of individual $\mathrm{i}$ reproduce themselves in the next generation is $p_{i}=f_{i} / \sum f_{i}$. Define select 
probabilities as the ratio of individual fitness value and population fitness value. The individual would be copied according to the selection probability. Individuals with bigger selection probability will be copied repeatedly in a higher opportunity. However, individuals with smaller selection probability are prone to be eliminated. In order to speed up the convergence, we take elite reserved strategy as select operator, only the best individual chromosome accounts for $5 \%$ of the total population quantity is reserved to the next generation population.

\subsection{Crossover}

Crossover namely chromosome population exchange some segments of two individual randomly, and create new individual. Crossover operator mainly includes partially mapped crossover, order crossover cross, cycle crossover and position based cross. This study, we use mapped crossover operator which can be described as follows. Select two chromosome segments with the same length from two intersecting father body randomly, cross the same position in the segment point and form mapping relation. For example, father generation individuals are 1-2-3- [4-5-6-7] -8-9 and 4-5-2- [1-8-7-6] -9-3, Cross the above segments in the '[ ]', Mapping relations are $4 \leftrightarrow 1,5 \leftrightarrow 8,6 \leftrightarrow 7$ and $7 \leftrightarrow 6$. First to exchange the cross segments, then, fill the father generation with points which are not conflict with the cross clips. As for the cross point which is conflict with the father generation, it must be filled with corresponding point according to the mapping relationship until there is no conflict point. For instance, father generation individuals 1-2-3- [4-5-6-7] -8-9 and 4-5-2- [1-8-7-6] -9-3 are changed into 1-2-3- [1-8-7-6] -8-9 and 4-5-2- [4-5-6-7] -9-3 after cross operation. Take the individual of 1-2-3[1-8-7-6] -8-9 for example, conflict points are 1 and 8. The offspring chromosomes is 4-2-3-1-8-7-6-5-9, after filling into corresponding point according to the mapping relationships of $4 \leftrightarrow 1$ and $5 \leftrightarrow 8$.

\subsection{Chromosome Aberration}

Chromosome aberration is means that some chromosomes are changed into new individuals because some positions of some chromosomes in the chromosomes population are mutated. Chromosome aberration operator consists of inversion aberration, insertion aberration, translocation aberration, interchange aberration, and so on. This study, we use inversion aberration which can be described as follows. First of all, select two truncation points in father body randomly, then, doing reverse sequence operation between the above truncation points. For instance, a chromosome 1-2- [3-4-5-6] -7-8-9, it's truncation marks are '[' and ']'. The offspring chromosome will changed into 1-2-6-5-4-3-7-8-9, when the substring sequence 3-4-5-6 is reversed as 6-5-4-3.

\section{Numerical Example}

To demonstrate the feasibility of the models and algorithm proposed in this study, we use Mat lab language to do two numerical examples. This section, we provide two numerical example results and their analysis.

\subsection{Initialization Optimal Route}

Some assumptions are set in this case study which can be described as follows.

Assumption 1: There are three distribution vehicles in the car yard, and the maximum load capacity of each vehicle is $12 \mathrm{~T}$. Vehicle speed is 1 unite, unloading time at the delivery points is neglected, each vehicle starts to distribute with full load.

Assumption 2: To simplify calculation, we assume that the distance between customer demand points and the distance between vehicle depots and customer demand points are both straight line, furthermore, all the above distance can be calculated according to the coordinates of customer demand points and logistics distribution center.

Assumption 3: The service priorities of customers only have two types, which are high and low. Customers with higher service priority have an infinite delay weight. Data about vehicle depots and customer demand points are shown in table 1. 
Table 1. Data about distance, demand and time windows

\begin{tabular}{|c|c|c|c|c|c|c|}
\hline \multirow{2}{*}{$\begin{array}{l}\text { Customer } \\
\text { Number }\end{array}$} & \multirow{2}{*}{$\begin{array}{l}\text { Horizontal } \\
\text { ordinate }(X) \\
\text { km }\end{array}$} & \multirow{2}{*}{ 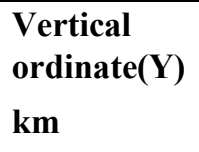 } & \multirow{2}{*}{$\begin{array}{l}\text { Customer } \\
\text { demand } \\
\mathrm{t}\end{array}$} & \multicolumn{2}{|c|}{ Time window(s) } & \multirow{2}{*}{$\begin{array}{l}\text { Service } \\
\text { priority }\end{array}$} \\
\hline & & & & $\begin{array}{l}\text { Upper } \\
\text { limit }\end{array}$ & $\begin{array}{l}\text { Lower } \\
\text { limit }\end{array}$ & \\
\hline 0 & 50 & 50 & - & - & - & - \\
\hline 1 & 60 & 40 & 1.9 & 140 & 200 & low \\
\hline 2 & 75 & 20 & 0.5 & 80 & 160 & low \\
\hline 3 & 60 & 0 & 1.2 & 100 & 130 & high \\
\hline 4 & 55 & 15 & 1.0 & 90 & 150 & low \\
\hline 5 & 40 & 40 & 1.8 & 120 & 180 & low \\
\hline 6 & 95 & 25 & 1.5 & 30 & 90 & low \\
\hline 7 & 30 & 30 & 0.9 & 90 & 150 & high \\
\hline 8 & 30 & 15 & 0.1 & 60 & 120 & high \\
\hline 9 & 15 & 5 & 1.6 & 30 & 90 & low \\
\hline 10 & 100 & 35 & 0.9 & 40 & 100 & low \\
\hline 11 & 10 & 20 & 1.2 & 30 & 90 & low \\
\hline 12 & 85 & 75 & 1.6 & 30 & 90 & high \\
\hline 13 & 15 & 45 & 1.8 & 0 & 60 & low \\
\hline 14 & 80 & 50 & 1.5 & 0 & 60 & low \\
\hline 15 & 80 & 10 & 0.4 & 50 & 110 & low \\
\hline 16 & 55 & 70 & 0.8 & 0 & 60 & low \\
\hline 17 & 5 & 95 & 1.9 & 150 & 210 & low \\
\hline 18 & 45 & 85 & 1.8 & 120 & 180 & low \\
\hline 19 & 25 & 100 & 0.8 & 120 & 180 & high \\
\hline 20 & 10 & 60 & 1.8 & 200 & 260 & low \\
\hline 21 & 65 & 90 & 0.1 & 60 & 120 & low \\
\hline 22 & 100 & 100 & 0.7 & 60 & 120 & low \\
\hline 23 & 30 & 70 & 1.6 & 200 & 260 & low \\
\hline
\end{tabular}

Note: data form random number generators which is restricted by the above three assumptions

Parameter set of Genetic algorithm: population size $(\mathrm{N})$ is 200 , cross probability $\left(p_{\mathrm{x}}\right)$ is 0.75 , aberration probability $\left(p_{\mathrm{v}}\right)$ is 0.10 , genetic algebra (Gen) is 400 , and the keep probability of excellent chromosome is $10 \%$. After repeatedly experiment, the optimal routes of the vehicle routing problem with time window (VRPTW) are as follows.

$\mathrm{V}_{1}: 0 \rightarrow 14 \rightarrow 10 \rightarrow 6 \rightarrow 2 \rightarrow 15 \rightarrow 3 \rightarrow 4 \rightarrow 1 \rightarrow 0$

$\mathrm{V}_{2}: 0 \rightarrow 16 \rightarrow 12 \rightarrow 22 \rightarrow 21 \rightarrow 18 \rightarrow 19 \rightarrow 17 \rightarrow 20 \rightarrow 23 \rightarrow 0$;

$\mathrm{V}_{3}: 0 \rightarrow 13 \rightarrow 11 \rightarrow 9 \rightarrow 8 \rightarrow 7 \rightarrow 5 \rightarrow 0$.

The total route length is $582.58 \mathrm{~km}$.

\subsection{Single Vehicle Scheduling of Distribution Delay}

80 minutes later when vehicles leave from the distribution center, by estimation, the delay time of vehicle 1 is about 20 minutes. At this time, vehicle 1 is traveling between customer 6 and customer 2. (Already served for customer 14, 10 and 6, but not served for customer 2). When the above distribution delay happened, we set the distribution delay point as virtual point $P$. If vehicle 1 travel as the initial optimal route, which is $\mathrm{P} \rightarrow 2 \rightarrow 15 \rightarrow 3 \rightarrow 4 \rightarrow 1 \rightarrow 0$, then, the total distribution distance is $582.58 \mathrm{~km}$, total distribution time is 26 seconds, distribution delay time for important customers is 11 seconds, it takes up $6.7 \%(11 / 30=6.7 \%)$ of the time window. But if call the single vehicle optimization model, new distribution route of vehicle 1 is $\mathrm{P} \rightarrow 15 \rightarrow 3 \rightarrow 4 \rightarrow 2 \rightarrow 1 \rightarrow 0$, 
and then, the total distribution distance is $596.00 \mathrm{~km}$, total distribution delay time is 7 minutes, distribution delay time of important customers is 0 minute, and routes change is 3 .

\subsection{Multi Vehicle Scheduling of Distribution Delay}

80 minutes later when vehicles leave from the distribution center, by estimation, the delay time of vehicle 1 is about 30 minutes. At this time, vehicle 1 is traveling between customer 6 and customer 2 (already served for customer 14, 10 and 6, but not served for customer 2), and the distribution delay point is set as virtual point $\mathrm{P}_{1}$. Vehicle 2 is traveling between customer 9 and customer 8 (already served for customer 13, 11 and 9, but not served for customer 8), and the distribution delay point is set as virtual point $P_{2}$. Vehicle 3 is traveling between customer 12 and customer 22 (already served for customer 16 and 12, but not served for customer 22), and the distribution delay point is set as virtual point $\mathrm{P}_{3}$. When the above distribution delay happened, if vehicle 1 travel as the initial optimal route, which is $\mathrm{P} \rightarrow 2 \rightarrow 15 \rightarrow 3 \rightarrow 4 \rightarrow 1 \rightarrow 0$, then, the total distribution distance is $582.58 \mathrm{~km}$, total distribution time is 46 seconds, distribution delay time for important customers is 21 seconds, it takes up $70 \%(21 / 30=70 \%)$ of the time window. In this situation, if call single vehicle optimization model, the solution cannot ensure the distribution service level of some key customers like customer 3, then, call multi vehicle optimization model. And the new routes are as follow. Vehicle 1: P1 $\rightarrow 2 \rightarrow 15 \rightarrow 7 \rightarrow 5 \rightarrow 0$, vehicle 2: $\mathrm{P} 2 \rightarrow 8 \rightarrow 9 \rightarrow 3 \rightarrow 4 \rightarrow 1 \rightarrow 0$, vehicle 2 : No change. The total distribution distance of the above new routes is $632.61 \mathrm{~km}$, total distribution delay time is 2 minutes, distribution delay time of important customers is 0 minute, and routes change is 2 .

5.4 Results Analysis

Table 2. Optimization results contrasts

\begin{tabular}{|c|c|c|c|c|c|}
\hline & \multirow[t]{2}{*}{ Contrast } & \multirow{2}{*}{$\begin{array}{l}\text { Total } \\
\text { distribution } \\
\text { distance } \\
\text { km }\end{array}$} & \multicolumn{2}{|c|}{$\begin{array}{l}\text { Time window deviate } \\
\text { (minute) }\end{array}$} & \multirow{2}{*}{$\begin{array}{l}\text { Route } \\
\text { deviation } \\
\text { number }\end{array}$} \\
\hline & & & $\begin{array}{l}\text { Total } \\
\text { customer }\end{array}$ & $\begin{array}{l}\text { Important } \\
\text { customer }\end{array}$ & \\
\hline \multirow[t]{2}{*}{$\begin{array}{l}\text { Single } \\
\text { vehicle }\end{array}$} & $\begin{array}{l}\text { (1)results that distribute as the } \\
\text { initial routes }\end{array}$ & 582.58 & 26 & 11 & 0 \\
\hline & $\begin{array}{l}\text { (2)optimization results of single } \\
\text { vehicle disruption management }\end{array}$ & 596.00 & 7 & 0 & 3 \\
\hline \multirow[t]{2}{*}{$\begin{array}{l}\text { Multi } \\
\text { vehicle }\end{array}$} & $\begin{array}{l}\text { (1)results that distribute as the } \\
\text { initial routes }\end{array}$ & 582.58 & 46 & 21 & 0 \\
\hline & $\begin{array}{l}\text { (2)optimization results of multi } \\
\text { vehicle disruption management }\end{array}$ & 632.61 & 2 & 0 & 2 \\
\hline
\end{tabular}

As is shown in table 2, as for the optimization results of single vehicle disruption management, it indicates an increase of the total distribution distance about 1.6\% ((592-582.58)/582.58) as compared with the results of the initial distribution routes. On the contrary, distribution delay time dropped $73 \%((26-7) / 26)$, especially, the distribution delay time of important customers is reduced to 0 . As for or the optimization results of multi vehicle disruption management, the total distribution distance increases about 8.59\% ((632.61-582.58)/582.58) as compared with the results of the initial distribution routes. But the distribution delay time drops $95.65 \%$ ((46-2)/46), especially, the distribution delay time of important customers is also reduced to 0 . With the application of single vehicle optimization model and multi vehicle optimization model, the total distribution distance has a slightly increase, but the distribution delay time especially for the key customers can be controlled effectively.

\section{Conclusions}

This study discusses the problem of distribution delay based on disruption management idea, and puts forward an idea that different customer has different service priority when distribution delay happened in the distribution network, then, constructed a single vehicle disruption management model with service priority and a multi vehicle disruption management model of distribution delay with service priority. These two modes will be called to ensure key customers service level when distribution delay happened. At the same time, these two models can be solved quickly by the improved Genetic Algorithm which is designed in this study.

To demonstrate the feasibility and validity of the models and algorithm proposed in this study, we make a 
numerical experiment. The numerical experiment result illustrate that call one of the two models to solve distribution delay problem can reduce all the key customers' distribution delay time to 0 even reduce the total distribution delay time of all the customers, in spite of a slight increase of total distribution distance. The models and algorithm is helpful for suppliers to improve their service level, especially to ensure key customers' service level when distribution delay happened.

\section{Acknowledgements}

This research is supported by National Natural Science Foundation of China (No.71201045), and Doctor Fund of Hefei University of Science \& Technology (NO.2011HGBZ1296) The authors also gratefully acknowledge the helpful comments and suggestions of the reviewers, which have improved the presentation.

\section{References}

Carlton, W. B., \& Barnes, J. W. (1996). Solving the Traveling Salesman Problem for the Vehicle Routing Problem with Time Windows. Operations Research, 40, 342-354.

Cher, A., \& Li, M. L. (2006). Concepts of disruption management, Crisis Management and Emergency management. Transactions of Emergency Management, 1(1), 8-9.

Clausen et a1. (2001). Disruption management operations research between planning and execution. $O R / M S$, $8(5), 40-43$.

Dantzig, G., \& Ramser, J. (1959). The truck dispatching problem. Management Science, 6, 80-91. http://dx.doi.org/10.1287/mnsc.6.1.80

Dror, M., Laporte, G., \& Trudeau, P. (1989). Vehicle Routing with Stochastic Demands: Properties and Solution Frameworks. Transportation Science, 23(1), 166-176. http://dx.doi.org/10.1287/trsc.23.3.166

Giaglis, G. M., Minis, I., \& Tatarakis, A. (2004). Minimizing Logistics Risk through Real-time Vehicle Routing and Mobile Technologies-Research to Date and Future Trends. International Journal of physical Distribution \& Logistics Management, 4(9), 749-764. http://dx.doi.org/10.1108/09600030410567504

Goetschalckx, M., \& Jacobs-Blecha, C. (1989). The vehicle routing problem with backhauls. European Journal of Operational Research, 42, 39-51. http://dx.doi.org/10.1016/0377-2217(89)90057-X

Golden, B., Assad, A., Levy, L., \& Gheysens, E. (1984). The fleet size and mix vehicle routing problem. Computers \& Operations Research, 1, 49-66. http://dx.doi.org/10.1016/0305-0548(84)90007-8

Huisman, D., Freling, R., \& Wagelmans, A. P. M. (2004). A Robust Solution Apporach to the Dynamic vehicle Sheduling Porhlem. Transportation Science, 38(4), 447-458. http://dx.doi.org/10.1287/trsc.1030.0069

Potvin, J.-Y., Xu, Y., \& Benyahia, J. (2006). Vehicle Routing and scheduling with Dynamic Travel Times. Computers \& operations Research, 33(4), 112 9-1137.

Taillard, E., Laporte, G., \& Gendreau, M. (1996). Vehicle Routing with Multiple Use of Vehicles. Journal of the Operational Research Society, 47, 1065-1070.

Toth, P., \& Vigo, D. (2002). The vehicle routing problem. American: Society for Industrial and Applied Mathematics, 29-31.

Van Woensel et al. (2008). Vehicle routing with dynamictravel times: a queuing approach. European Journal of Operational Research, 186(3), 990-1007. http://dx.doi.org/10.1016/j.ejor.2007.03.012

Wang, X. P., Niu, J., \& Hu, X. P. (2007). Rescue Strategies of the VRPTW Disruption. System Engeering theory \& Practice, 12, 104-110.

Wang, X. P., Wang, C., \& Hu, X. P. (2008). Study on Disruption Management of Vehicle Routing Problem with the Changes of Time Windows and Delivery Weight of Customers. Management Science, 21(5), 110-120.

Yang, W. Z., Wang, Z., \& Hu, X. P. (2010). Disruption management model and algorithm for urban distribution with travel time delay. Computer Integrated Manufacturing Systems, 16(2), 331-339.

Yu, G., \& Qi, X. T. (2004). Disruption management: framework, models and applications. Singapore: World Scientific Publishing Co. Pte. Ltd.

Zeimpekis, V., Giaglis, G. M., \& Minis, I. (2005). A Dynamic Real-time Fleet Management System for Incident Handling in City Logistlcs. Vehicular Technology Conference, 2900-2904.

Zeng, A. Z., Durach, C. F., \& Fang. Y. (2012). Collaboration decisions on disruption recovery service in urban 
public tram systems. Transportation Research Part E-Logistics and Transportation Review, 48(3), 578-590. http://dx.doi.org/10.1016/j.tre.2011.11.005

Zhang, X., \& Pan, S. F. (2008). A Disruption Management Model of Logistic Distribution Delay. System Engineering, 10(10), 13-16.

Zhang, Y. (2007). Disruption Management Model of Delaying Problem in Logistics Distribution. Dalian, Dalian University of Technology.

Zhang, Y. H. (2005). Study of Commercial Vehicle Emergent Dispatch. Beijing, Beijing University of Technology. 